\title{
Hydroxykynurenine/hydroxyanthranilic acid ratios and febrile convulsions
}

\author{
J MCKIERNAN, D MELLOR, S COURT, J EDSON, AND K LACEY \\ Department of Child Health and University Hospital, Queen's Medical Centre, Nottingham
}

SUMMARY Hydroxykynurenine/hydroxyanthranilic acid ratios were measured in children with febrile convulsions, afebrile fits, and fever, as well as in healthy controls. Increased ratios were found not only in the children who had fits but also in the children who were febrile and did not have fits. It is suggested that a raised hydroxykynurenine/hydroxyanthranilic acid ratio does not necessarily indicate vitamin B6 deficiency but may represent a nonspecific response of tryptophan metabolism to stress.

Febrile convulsions are a common problem in children, affecting between 2 and $5 \%$ in the 6 -month to 5-year age range. ${ }^{1}$ The pathogenesis of these seizures is imperfectly understood. The elucidation of the syndromes of vitamin B6 deficiency and dependency in which fits are a major clinical feature, raised the possibility that a specific biochemical basis might also be found for the more common convulsive disorders of childhood. The trytophan load test has been traditionally regarded as a test of vitamin B6 status although it is now appreciated that other influences-such as oestrogenic and corticosteroid hormones, stress, and the general state of protein metabolism-may affect the result. ${ }^{2}$ Tryptophan load tests were carried out on 20 children with febrile convulsions by Carredu et al. ${ }^{3}$ and abnormalities in metabolite excretion were found. It was therefore suggested that a relationship exists between febrile convulsions and pyridoxine deficiency. Lennox-Buchthal ${ }^{4}$ suggested that the association betweeen vitamin B6 and febrile convulsions be further explored, noting that in the syndrome of vitamin B6 dependency, fever increases the need for pyridoxine and may precipitate convulsions.

Tryptophan load tests in the past have mainly been carried out using timed collections of urine after the tryptophan load, and then by measuring a number of urinary metabolites. Such collections are impractical in many children. In addition, great variability in metabolite excretion is found. We used a modification of the tryptophan load test, and measured only two urinary metabolites-hydroxykynurenine (HK) and hydroxyanthranilic acid (HA), which were then expressed as a ratio- $\mathrm{KH} / \mathrm{HA}$. This ratio is thought

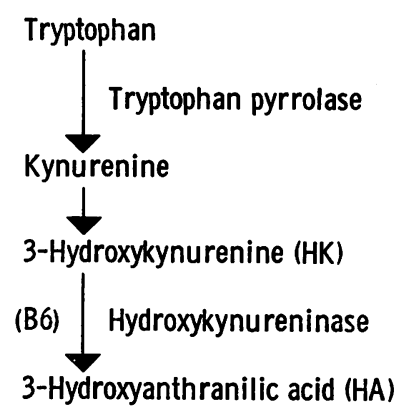

Fig. 1 Major pathway of tryptophan catabolism. B6 indicates the pyridoxal-phosphate-dependent enzyme reaction.

to reflect the activity of the liver enzyme kynureninase, which requires vitamin $\mathrm{B} 6$ as a cofactor (Fig. 1). It is considered to be a reliable index of pyridoxine deficiency ${ }^{5}$ and, because it is a ratio, it has the advantage of not requiring a carefully timed urine collection. We estimated HK/HA ratios after a tryptophan load in normal children, children who were febrile, children who had afebrile fits, and children with febrile convulsions, and report our results.

\section{Patients and methods}

Each of the 69 children with febrile convulsions had been admitted to hospital after a first or second febrile fit. Ages ranged between 6 months and 5 years. The diagnosis was based on the usual criteria-that 
is, history of a convulsion associated with a temperature of $37.5^{\circ} \mathrm{C}$ or greater. Fits were regarded as complex in 23 cases - prolonged, lasting longer than 15 minutes (17 cases), multiple (6 cases), or focal ( 2 cases). Children with central nervous system infection or diseases were excluded. Six children aged between 1 and 4 years who had been admitted to hospital with febrile illnesses constituted the 'fever only' group. Six children, aged between 2 and 5 years who had a first nonfebrile fit, who were otherwise normal and not on anticonvulsant treatment, comprised the 'afebrile fit' group. Seven healthy children, who were not in hospital, acted as controls.

Tryptophan load test. Tryptophan powder $100 \mathrm{mg} / \mathrm{kg}$ body weight was given as a sweetened suspension. A single urine specimen was collected at least 20 minutes after the ingestion of the dose and frozen until assay.

Measurement of HK and HA. Fractionation of tryptophan metabolites from urine was carried out by an ion exchange resin technique based on the methods of Brown and Price. ${ }^{6}$ Urine samples were hydrolysed using the method described by Heeley. ${ }^{7}$ The eluted HK and HA were measured spectrophotometrically using Brown's method. ${ }^{8} \mathrm{~A}$ blank and a duplicate test were performed on each sample. Column recoveries were carried out with each batch of tests and the recovery of metabolites added to urine samples was also determined; recovery was $89 \% \pm 7$ (mean $\pm \mathrm{SD}$ ) for both HK and HA.

Plasma cortisol. Blood samples were collected from 15 children with febrile convulsions at the time of admission to hospital and plasma cortisol was measured using a solid phase radioimmunoassay supplied by the Tenovus Institute, Cardiff.

All results were analysed by Student's $t$ test for unpaired samples.

\section{Results}

The $\mathrm{HK} / \mathrm{HA}$ ratio in the healthy control children was $0.79 \pm 0.16$ (mean \pm SE) (Fig. 2). Ratios were significantly higher $(P<0.05)$ in the groups of children with fever, afebrile fits, and febrile convulsions. The majority of children with febrile convulsions had raised $\mathrm{HK} / \mathrm{HA}$ ratios, but no relationship between $\mathrm{HK} / \mathrm{HA}$ ratio and age, sex, height of fever, or complexity of fit could be found.

In 30 cases it was possible to relate the $\mathrm{HK} / \mathrm{HA}$ ratio to the time interval between the convulsion and the tryptophan load test (Fig. 3). Increased ratios

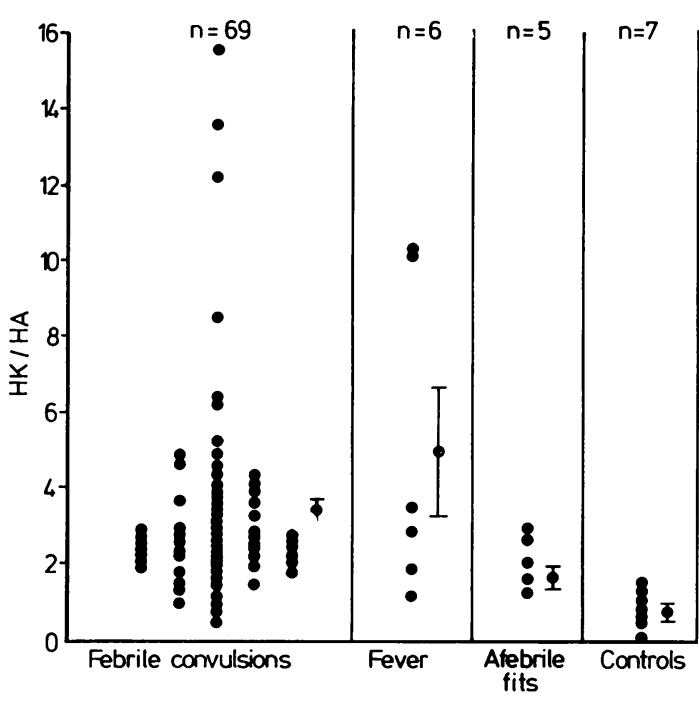

Fig. $2 \mathrm{HK} / \mathrm{HA}$ ratios in the four groups of children. Solid lines indicate means $\pm S E$.

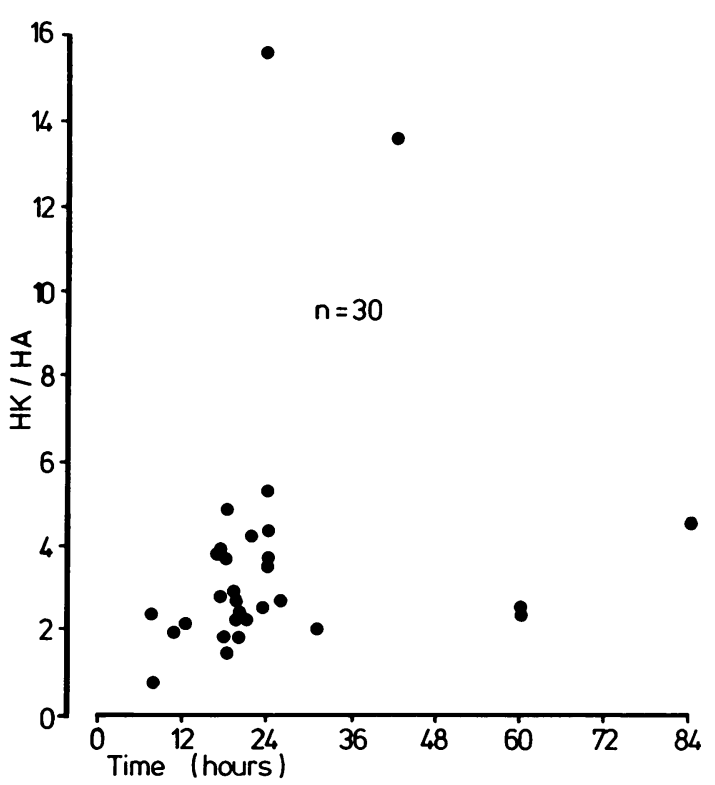

Fig. $3 \mathrm{HK} / \mathrm{HA}$ ratios in relation to time after the fit.

were found up to 4 days after the fit. In 6 cases the tryptophan load test was repeated one month or more after the convulsion, when the child was well. Ratios in the normal range were found in 4 children and in 2 values were still raised. We found no relationship between the HK/HA ratios and plasma cortisol in 15 children with febrile convulsions. 


\section{Discussion}

This study shows that in a small group of healthy children, HK/HA ratios after a tryptophan load were within the range found in healthy women; ${ }^{9}$ we could not find any results for healthy children. From our results it is also clear that febrile illnesses, not accompanied by convulsions, may induce a rise in the HK/HA ratio. Even in a child who had had a single nonfebrile fit, the ratio was higher than in the control.

We have confirmed therefore, that tryptophan metabolism is disordered in children with febrile convulsions as was suggested by Carredu et al. ${ }^{3}$ Interestingly, Rabe and Plonko ${ }^{10}$ found abnormal xanthurenic acid excretion after a tryptophan load in a small number of children with infections, some of whom had febrile fits. None showed evidence of pyridoxine depletion on the basis of the 4-pyridoxic acid excretion test.

Despite the refinements in the tryptophan load test, we feel that an abnormal $\mathrm{HK} / \mathrm{HA}$ ratio is not a specific test of pyridoxine deficiency. Coon and Nagler ${ }^{2}$ stressed that the test might be influenced by other factors-such as stress, adrenal glucocorticoids, sex hormones, and the general state of protein metabolism. Increased hormone activity, as happens in stress, and increased protein catabolism may increase the flow of metabolites down the kynurenine pathway and result in raised HK/HA ratios even in the presence of normal amounts of pyridoxine (Fig. 1). We feel that it is likely that some such mechanism may underlie the increased HK/HA ratios that we have found in children with fever, fits, and febrile convulsions although we were unable to demonstrate any relationship between plasma cortisol levels and the HK/HA ratios.

Despite these considerations, our results do not exclude the possibility that fever or infection can induce a transient state in which vitamin B6 demand may exceed supply. Such a state of relative B6 deficiency could conceivably be the underlying mechanism in the pathogenesis of febrile convulsions. If so, this would have clear implications for prophylaxis in children after an initial febrile convulsion.
We thank the nursing staff of Princess Mary ward, Nottingham Children's Hospital, for carrying out the tryptophan load tests, our colleagues for allowing us to study patients under their care, Professor D Hull for support, and Mr D Henson, Department of Clinical Chemistry, University Hospital, Nottingham, for performing the plasma cortisol radioimmunoassays.

Financial support was provided by the Nottingham Medical School Trust Fund.

\section{References}

1 Lennox-Buchthal M A. A summing up: clinical session. In: Brazier M A B, Coceani F, eds. Brain dysfunction in infantile febrile convulsions. New York: Raven Press, 1976: 327-51.

2 Coon W W, Nagler E. The tryptophan load as a test for pyridoxine deficiency in hospitalized patients. Ann $N Y$ Acad Sci 1969; 166: 30-43.

3 Carredu P, Appollonio I, Giovannini M. Troubles du métabolisme du tryptophane dans le méchanisme pathogénique des convulsions hyperpyrétiques. Pediatrie 1962; 17: 359-72.

${ }^{4}$ Lennox-Buchthal M A. Febrile convulsions, a reappraisal. Amsterdam: Elsevier, 1973.

5 O'Brien D, Jensen C B. Pyridoxine dependency in two mentally retarded subjects. Clin Sci $1963 ; 24$ : 179-86.

6 Brown R R, Price J M. Quantitive studies on metabolites of tryptophan in urine of dog, cat, rat, and man. J Biol Chem 1956; 219: 985-97.

7 Heeley A F. The effect of pyridoxine on tryptophan metabolism in phenylketonuria. Clin Sci $1965 ; 29$ : 465-73.

8 Brown R R. The isolation and determination of urinary hydroxykynurenine. J Biol Chem 1957; 227 : 649-52.

9 Adams P W, Rose D P, Folkard J, Wynn V, Seed M, Strong R. Effect of pyridoxine hydrochloride (vitamin B6) upon depression associated with oral contraception. Lancet 1973 ; i: 897-904.

10 Rabe E F, Plonko M. Pyridoxine hydrochloride (vitamin B6) need in infants and children. Am J Dis Child 1956; 92: 382-9.

Correspondence to Dr J McKiernan, Paediatric Unit, Limerick Regional Hospital, Dooradoyle, Limerick, Eire.

Received 4 October 1979 